\title{
SAFETY OF ORAL NIFUROXAZIDE - ANALYSIS OF DATA FROM A SPONTANEOUS REPORTING SYSTEM
}

\author{
KATARZYNA KARŁOWICZ-BODALSKA ${ }^{1 *}$, KRYSTYNA GŁOWACKA ${ }^{2}$, \\ KAMILA BOSZKIEWICZ ${ }^{3}$, STANISŁAW HAN ${ }^{1}$ and ANNA WIELA-HOJEŃSKA ${ }^{2}$
}

\author{
${ }^{1}$ Department of Industrial Pharmacy, Wroclaw Medical University, \\ Faculty of Pharmacy, ul. Borowska 211, 50-556 Wrocław, Poland \\ ${ }^{2}$ Department of Clinical Pharmacology, Wroclaw Medical University, \\ Faculty of Pharmacy, ul. Borowska 211, 50-556 Wrocław, Poland \\ ${ }^{3}$ Pharmaceutical Production Company PPF Hasco-Lek S.A. Wroclaw, \\ ul. Żmigrodzka 242E, 51-131 Wrocław, Poland
}

\begin{abstract}
Nifuroxazide is a popular chemotherapeutic agent, that is widely used in Poland in acute and chronic bacterial diarrhea treatment. It is available in pharmacies as over-the-counter tablets for adults and a suspension on prescription for children. The aim of the study was to assess the safety of nifuroxazide therapy. Adverse reaction reports from the Regional Pharmacovigilance Center in Wroclaw, the pharmaceutical company PPF Hasco-Lek, and VigiAccess and EudraVigilance databases were analyzed. Based on the analysis of the data collected from the above sources, nifuroxazide have shown a high therapeutic value in the gastrointestinal tract infections, maintaining high safety of usage at the same time. The number of drug application adverse reactions in Poland is not so high primarily due to high safety profile and low patient awareness of the possibility of reporting drug side effects.
\end{abstract}

Keywords: diarrhea, gastrointestinal tract, pharmacovigilance, drug interactions, nifuroxazide

Nifuroxazide (4-hydroxy-benzoic acid; 2-[(5nitro-2-furanyl)methylene]hydrazide) is a chemotherapeutic agent belonging to the nitrofuran family. Indications for its use are acute and chronic bacterial diarrhea and other diseases with diarrhea (1). The drug has local antibacterial activity against some Gram-positive bacteria of the genus Staphylococcus and Gram-negative bacteria from the Enterobacteriaceae family: Yersinia spp., Escherichia spp., Enterobacter spp., Klebsiella spp. and Salmonella spp. It does not affect the bacteria Proteus vulgaris, Proteus mirabilis and Pseudomonas aeruginosa (13). Recently, attention has been paid to its antitumor potential in neuroblastoma, multiple myeloma, colorectal and breast cancer, efficacy in the treatment of graft-versus-host disease and nephroprotection in diabetes (4-10).

The exact mechanism of action of nifuroxazide has not yet been identified. It probably has an inhibitory effect on dehydrogenase activity and protein synthesis in bacterial cells. Aldehyde dehydrogenase $(\mathrm{ALDH})$ is responsible for the bioactivation of this prodrug. It has been found that nifuroxazide is a potent inhibitor of the Janus kinase 2 pathways and transcription factor 3 (JAK2/STAT3). It also has direct effects on oxidative stress and apoptosis (2, 4-6).

Frequent self-administration of this drug by patients makes it necessary to pay attention to selfmedication, which in accordance with the guidelines of the World Health Organization is defined as "using medication by the consumer to treat signs/symptoms or minor health problems, recognized as such by themselves". In practice, this term also includes treatment by family members or friends, mainly, when the child is being treated (11). It should be responsible and safe, especially considering that diarrhea, in particular, acute diarrhea, is a serious problem of primary and hospital care. It's serious complications, such as electrolyte disturbances, oligovolemic shock and even death, are dangerous especially for newborns, babies, small children and elderly people, due to their susceptibility to dehydration $(12,13)$. The fact that adverse effects inevitably accompany the desired therapeutic effects

\footnotetext{
* Corresponding author: e-mail: katarzyna.karlowicz-bodalska@umed.wroc.pl
} 
of medical products cannot be ignored. The available data shows how important this problem is. Drug-related complications affect $3.7 \%$ to $38 \%$ of patients. Kampichit et al. found that the most commonly reported by patients adverse reactions are these related to skin and subcutaneous tissue disorders, gastrointestinal disorders and nervous system disorders (14). According to Pirmohamed et al., involving 18820 patients in England, adverse-drug reactions are directly responsible for $6.5 \%$ of all admissions to hospitals (15). Numerous studies indicate that many drug problems can be prevented by, among others, individualization and personalization of therapy, assessment of their safety profile, as well as pharmacovigilance.

All the above-mentioned issues were an inspiration to undertake research, aimed at assessing reports of adverse effects of nifuroxazide therapy from various sources.

\section{EXPERIMENTAL}

The study material consisted of reports of adverse effects of nifuroxazide, obtained from the Regional Pharmacovigilance Center at the Department of Clinical Pharmacology at Wroclaw Medical University (2005 - September 2018), from a producer of medical products with nifuroxazide, PPF Hasco-Lek S.A. (2007 - September 2018), as well as the EudraVigilance and VigiAccess databas- es (1980 - September 2018). In addition, a systematic review of the literature from the years 19782018, derived from PubMed, Embase, CINAHL, Scopus, Micromedex, and Cochrane Library, relating to pharmacovigilance in the context of diarrhea was performed. Ethics approval was not required for this study according to Polish regulations (16).

\section{RESULTS AND DISCUSSION}

In the years 2006 - September 2018, one report of nifuroxazide adverse reaction was received by the Regional Pharmacovigilance Center in Wroclaw. The complication was observed in a six-year-old boy with positive family and individual history of atopic diseases, treated in hospital due to pneumonia with undetermined etiology. The patient received polytherapy, including nifuroxazide and medications such as human immunoglobulin-Pentaglobin, ceftazidime-Biotum, budesonidum-Pulmicort, salbutamol-Ventolin, promethazini hydrochloridum-Diphergan, hydrocortisone-Hydrocortyzon, salmeterolum-Serevent, rovamycine-Rovamycin, and Aminopterin. Two minutes after administration of Pentaglobin in a slow infusion, the patient had anxiety, apnea, and bruising of the lips. After applying the adrenaline, the symptoms subsided. In this case, human immunoglobulin Pentaglobin was responsible for the occurrence of side effects. There was a recovery without lasting consequences.

Table 1. Analysis of the frequency of adverse effects of nifuroxazide reported to the VigiAccess database in the period 1980 - September 2018 .

\begin{tabular}{|l|c|c|}
\hline \multicolumn{1}{|c|}{ ADRs } & Number of applications & Percentage $(\%)$ \\
\hline Skin and subcutaneous tissue disorders & 332 & 59.4 \\
\hline General disorders and administration site conditions & 69 & 12 \\
\hline Gastrointestinal disorders & 68 & 6 \\
\hline Blood and lymphatic system disorders & 35 & 6.1 \\
\hline Nervous system disorders & 34 & 4.7 \\
\hline Immune system disorders & 26 & 4.5 \\
\hline Respiratory, thoracic and mediastinal disorders & 25 & 3.9 \\
\hline Injury, poisoning and procedural complications & 22 & 3.6 \\
\hline Hepatobiliary disorders & 20 & 3.4 \\
\hline Investigations & 19 & 2.7 \\
\hline Renal and urinary disorders & 16 & 2.7 \\
\hline Vascular disorders & 15 & 2.5 \\
\hline Musculoskeletal and connective tissue disorders & 14 & 2.5 \\
\hline Psychiatric disorders & 14 & 2.2 \\
\hline Eye disorders & 12 & \\
\hline
\end{tabular}




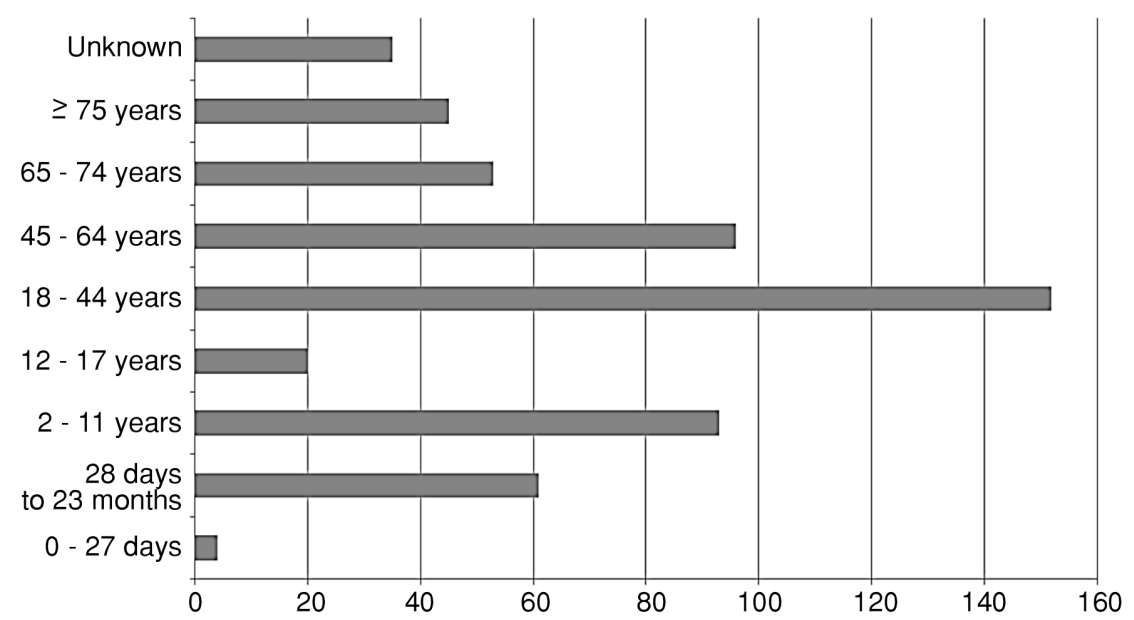

Figure 1. Analysis of the age of patients who experienced adverse effects of nifuroxazide reported to the VigiAccess database (period 1980 - September 2018)

The pharmaceutical company PPF Hasco-Lek S.A., a producer of medications containing nifuroxazide, both available over the counter (OTC) Nifuroksazyd Hasco (coated tablets, $100 \mathrm{mg}$ nifuroxazide), Nifuroksazyd 200 Hasco (coated tablets, $200 \mathrm{mg}$ nifuroxazide) - and available on prescription - oral suspension Nifuroksazyd Hasco (220 mg per $5 \mathrm{~mL}$ ) - in the period 2006 to September 2018, registered 4 reports of adverse reactions to nifuroxazide. The first of them was recorded in 2007. It concerned a four-year-old boy who had nausea and vomited after 15 min after administration of nifuroxazide. In 2010, information about the occurrence of an allergic reaction in the form of pruritus, burning, redness and rash, also in a child, after administration of nifuroxazide in oral suspension (220 mg per $5 \mathrm{~mL}$ ) was sent. These symptoms, despite treatment with antiallergic clemastine, persisted for a week. The next report of adverse effects was submitted in 2011. It was observed in a fiveyear-old boy after administration of nifuroxazide as an urticarial rash presented on the abdomen, chest, and back, which resolved after discontinuation of the drug. The only serious adverse effect of nifuroxazide was reported to the marketing authorization holder in 2012 and concerned a 14-month-old boy. After administration of nifuroxazide, the patient had massive urticaria covering about $30 \%$ of his body surface. In addition, swelling of the fingers, hands and mouth was observed. The patient required hospitalization.

Global and European Pharmacovigilance databases contain more information about the complica- tions of nifuroxazide therapy. The VigiAccess database has recorded 559 cases since 1980, including $58 \%$ from the last 10 years. Skin reactions (rash, pruritus, urticaria, angioedema, erythematous rash) and general disorders such as facial swelling, chills and malaise were the most commonly reported. Their complete analysis is presented in Table 1.

The age structure of patients who experienced adverse reactions of nifuroxazide is shown in Figure 1. The most reports were recorded in the following age groups: $18-44$ years - 29\%, 2-11 years - 17\%, 45-64 years $-17 \%$ and children up to 2 years old $-11 \%$.

The EudraVigilance database contained 142 cases of adverse reactions related to nifuroxazide therapy. Most reports concerned people aged between 18 and 64 (43\%). Mainly skin reactions, facial swelling, malaise, and gastrointestinal disorders were observed.

A review of the literature showed good tolerance of nifuroxazide treatment and in general, lack of complications during therapy. Only a few cases have been reported. The first concerned a 47-yearold female patient who came to the hospital with a very extensive rash on the hands and with severe pruritus. The interview showed that the patient was working in a pharmaceutical company in the production of nifuroxazide-containing medicines. The patient did not use protective gloves and the cause of the severe rash was a contact allergy to nifuroxazide (17). A case of a middle-aged patient was also described, in whom after taking two capsules of nifuroxazide $200 \mathrm{mg}$, typical symptoms of pancreatitis occurred (18). 
Nifuroxazide is an active substance available on the pharmaceutical market since 1964 when it was registered in France as the original product Ercerfuryl from Sanofi company. Later, nifuroxazide was introduced in other European countries, Africa, Asia, and Latin America. In Poland, products containing nifuroxazide have been on sale since 1990 and are available with a prescription as an oral suspension $(220 \mathrm{mg} / 5 \mathrm{~mL})$ and over the counter (OTC) in the form of tablets and capsules in doses of 100 and $200 \mathrm{mg}$. Annual sales of nifuroxazide are systematically growing - from 2.7 million packages in 2009 to over 3 million packages in the years 2015-2016. In 2017, it was at the level of 2.7 million packages (19). Sales data for nifuroxazide in Poland are shown in Figure 2.

Data obtained in our study confirm these observations. The Regional Pharmacovigilance Center in Wroclaw does not have any report in which a relationship between nifuroxazide and adverse effects can be found. Data obtained from the marketing authorization holder - the pharmaceutical company Hasco-Lek S.A. - indicate four reports sent in the last ten years (2007-2018), including one classified as severe due to the need to hospitalize the patient. In the period from 1980 to September 2018, the European database EudraVigilance and the WHO VigiAccess database received respectively 142 and 559 reports about adverse effects of nifuroxazide treatment. Most of them were related to gastrointestinal disorders and hypersensitivity reactions, such as rash, urticaria or angioedema.
A review of the literature indicates the efficacy of nifuroxazide, which used in the treatment of acute diarrhea, as well as in prophylaxis of traveler's diarrhea, significantly shortens the duration of symptoms, reduces excessive activity of the gastrointestinal tract and quickly restores normal stool consistency $(20,21)$. Herbert L. DuPont et al. reported its similar efficacy to rifaximin and ciprofloxacin (22). Despite reporting two cases of serious adverse reactions (severe contact allergy, acute pancreatitis), Kieć - Skwierzyńska et al. describe a clinical case in which a 47-year-old female was admitted to the hospital with a very extensive rash on the hands and accompanied by severe pruritus. The interview showed that she was an employee of a pharmaceutical company and worked on the production of nifuroxazide. The patient did not work in protective gloves. After pharmacotherapy, the symptoms subsided but returned after her back to work (17). The next described case concerned the middle-aged patient who had typical symptoms of pancreatitis after taking 2 capsules of nifuroxazide $200 \mathrm{mg}$ (18). Most publications indicate very good tolerance of nifuroxazide therapy $(20,21,23)$.

Nifuroxazide distinguishes itself from other nitrofuran derivatives. The drug has a local antibacterial effect, because as a practically insoluble substance, it occurs in the intestinal lumen, i.e. at the target site, at a significant concentration, and is practically not absorbed into the bloodstream. Thanks to this, systemic adverse effects are rarely experienced during its use. Nifuroxazide does not adversely affect the physi-

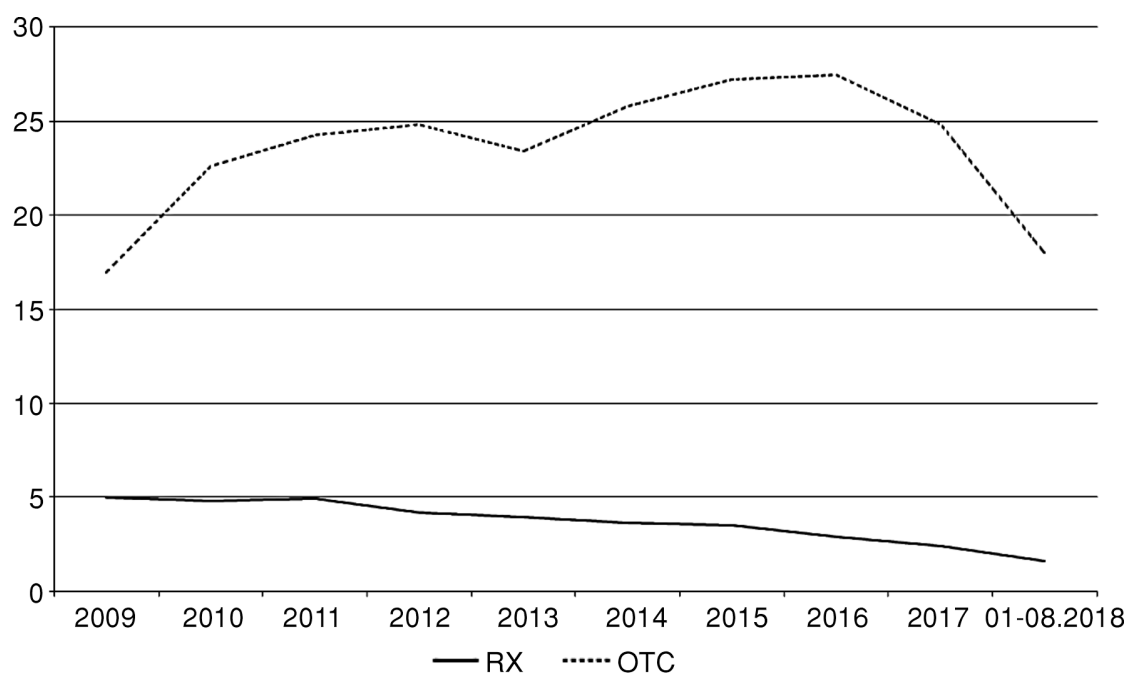

Figure 2. Analysis of sales of packaging containing nifuroxazide (in hundreds of thousands) in Poland (period 2009 - August 2018) (19) 


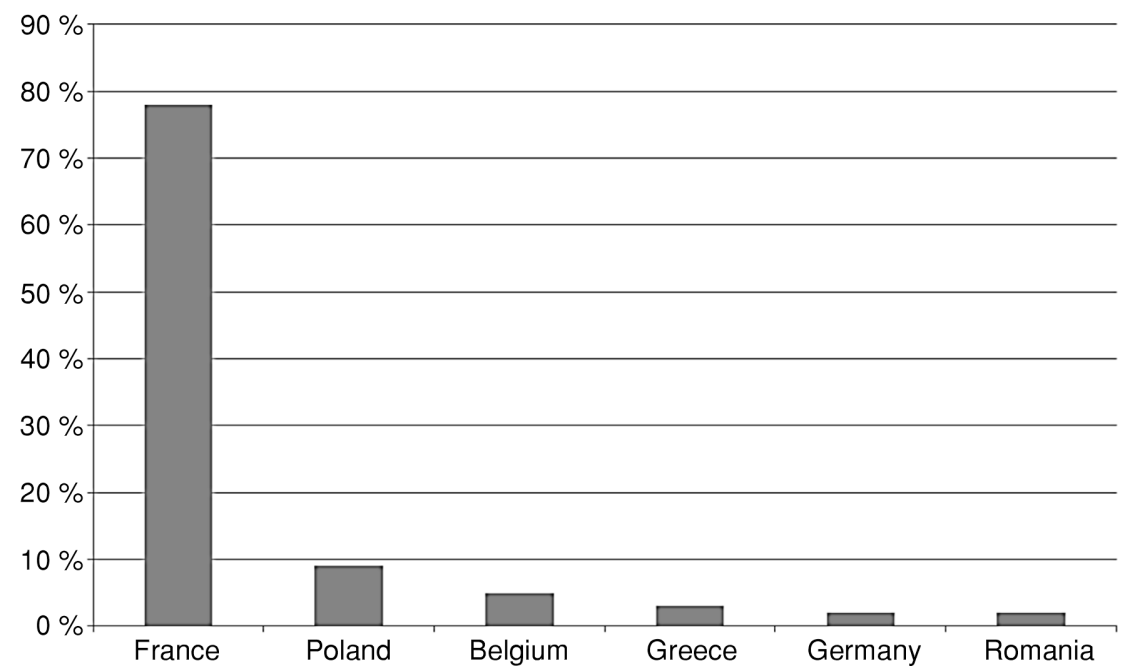

Figure 3. Analysis of percentage share of reports of adverse effects of nifuroxazide to EudraVigilance by country - period up to September $2018(27)$

ological intestinal bacterial flora, despite the broad spectrum of antibacterial activity $(1,24)$. Studies in healthy volunteers receiving $800 \mathrm{mg}$ or $1200 \mathrm{mg}$ of nifuroxazide per day for 6 days showed its safety and the lack of formation of resistant bacterial strains. Buisson et al. confirm also that nifuroxazide, even in high dosage, does not affect microbial intestinal ecosystem. Importantly, nifuroxazide treatment does not induce cross-resistance to other antibacterial agents, including quinolones and sulfonamides, which indicates the possibility of its use in combined therapy of intestinal infections (1-3, 23-24).

Among the adverse effects of nifuroxazide listed in the summary of the product, characteristics are hypersensitivity reactions manifested by dyspnea, swelling of the face, lips, and tongue, and pruritus. Their occurrence requires immediate discontinuation of the nifuroxazide. In rare cases $(=1 / 10000$ to $<$ $1 / 1000)$ skin reactions in the form of a rash are observed. There was also a case of nodular itching in the course of contact allergy to nifuroxazide. In addition, abdominal pain, nausea, worsening of diarrhea, and granulocytopenia have been reported (1-3).

The French safety assessment of nifuroxazide therapy is different, although the availability of products is similar in Poland - categories $\mathrm{Rp}$ and OTC. There is an opinion by publishers of the Prescrire International monthly, aimed at professional medical staff, that due to numerous reports of adverse effects and poorly documented efficacy, nifuroxazide should be withdrawn from the market (25). At the beginning of 2017, the French National
Agency for Medicines and Health Products Safety published a safety report for nifuroxazide $(25,26)$. The collected data covered the entire period of the drug's presence on the French market - from 1964 to March 2016. A total of 646 reports of adverse reactions were recorded, of which 288 were classified as severe. Among 97 serious immunoallergic reactions, there were 16 cases of anaphylactic shock and 41 of angioedema. Of the 61 serious skin side effects reported, 27 cases were related to severe skin reactions (including two cases of acute generalized exanthematous pustulosis and one case of toxic epidermal necrolysis). The only drug suspected of their induction was nifuroxazide. Other adverse reactions were associated with hematological disorders (42 cases), such as agranulocytosis, thrombocytopenia, hematological anemia, liver damage (24 reports), neurological disorders (17 reports), gastrointestinal disorders (14 reports) and kidney disorders (9 reports). The comparison of the number of French reports of adverse effects of nifuroxazide (646 cases up to 2016) and the data from the VigiAccess global database (559 up to September 2018) led to the conclusion that there was no reporting of all adverse reactions to the global database. In addition, it should be noted that the majority of cases of adverse effects of nifuroxazide reported to the EudraVigilance database come from France (78\%). The second country, in terms of the number of adverse effects of nifuroxazide reported to EudraVigilance, is Poland $(9 \%)$. The percentage share of reports from other countries is shown in Figure 3. 
Nifuroxazide is a popular drug in Poland and has been used for many years in the treatment of bacterial diarrhea. The availability of the drug has an impact on the increase in its sales, currently, oral tablets with 100 and $200 \mathrm{mg}$ of nifuroxazide, previously prescribed $\mathrm{Rx}$, are available without a prescription (OTC). Oral suspension of nifuroxazide in a dose of $220 \mathrm{mg} / 5 \mathrm{~mL}$ is still available for the prescription. The patient has the option of self-medication, so there may be risks associated with improper use or abuse which can lead to complications and consequently to hospitalization. Despite the increase in sold packaging per year, there is no significant number of reported adverse reactions in the assessed period. This may indicate a high safety profile of nifuroxazide therapy or low patient awareness of the possibility of reporting side effects. Recently nifuroxazide has been found to have anticancer activity in osteosarcoma, neuroblastoma, multiple melanomas, colon, breast cancer. Its properties are promising, such as inhibiting cell proliferation, inducing cell apoptosis, as well as impairing cell migration. It may attenuate graft versus host disease (GvHD) and improve the survival rate. It has a potential effect in terms of regulating $\mathrm{T}$ cell differentiation with a significant decrease in the secretion of IFN- $\alpha$ and IFN- $\gamma$ cytokines. In these activities, the blocking of the STAT3 pathways plays an important role. Nifuroxazide can be effective as prophylaxis or second-line therapy for aGvHD (4-8, 28$30)$. It can be assumed that this old drug will find a new application in medicine.

There are some limitations to our study. The main limitation is that our study material consisted of reports of adverse effects of nifuroxazide obtained from only one Regional Pharmacovigilance Center (Regional Pharmacovigilance Center at the Department of Clinical Pharmacology at Wroclaw Medical University) and only one producer of medical products with nifuroxazide (PPF Hasco-Lek S.A.). Despite these limitations, this study sheds new light on the safety of nifuroxazide therapy, especially that there is a lack of newly published data about safety.

\section{CONCLUSIONS}

Based on the literature data and the author's observations, a favorable safety profile of nifuroxazide should be stated. The use of this chemotherapeutic can, therefore, be a safe alternative to the treatment of bacterial infections of the gastrointestinal tract, especially at a time of increasing antibiotic resistance. Nifuroxazide can be used as a medi- cine of choice in the mild form of acute infectious diarrhea, instead of antibiotics, which are prescribed in more serious cases (31). In the case of a complicated form of acute diarrhea, combination therapy with antibiotic and nifuroxazide is indicated. At the same time, it is necessary to increase the awareness of patients and health care professionals about the monitoring of adverse drug reactions, which will undoubtedly contribute to the optimization of pharmacovigilance.

\section{REFERENCES}

1. Nifuroxazide - Summary of Product Characteristics, 2018 (accessed on 5.06.2018).

2. Podlewski J.K., Podlewska A: Leki Współczesnej Terapii. Wydanie XXII, Medical Tribune Polska 2019.

3. Fernandes M.B., Goncalves J.E., Scotti M.T., de Oliveira A.A., Tavares L.C., Storpirtis S.: Toxicol. In Vitro 26, 535 (2012).

4. Sarvi S., Crispin R., Lu Y., Zeng L., Hurley T. et al.: Cell Chem. Biol. 25, 1 (2018).

5. Zhao T., Jia H., Cheng Q., Xiao Y., Li M. et al.: Oncol. Rep. 37, 3405 (2017).

6. Ye T.H., Yang F.F., Zhu Y.X., Li Y.L., Lei Q. et al.: Cell Death Dis. 8, 1 (2018).

7. Zhu Y., Ye T., Yu X., Lei Q., Yang F. et al.: Sci. Rep. 6, 20253 (2016).

8. Jia H., Cui J., Jia X., Zhao J., Fenget Y. et al.: Mol. Med. Rep. 16, 9480 (2017).

9. Elsherbiny N.M., Zaitone S.A., Mohammad H.M.F., El-Sherbiny M.: Toxicol. Mech Method. 28, 467 (2018).

10. Said E., Zaitone S.A., Eldosoky M., Elsherbiny N.M.: Chem. Biol. Interact. 281, p 111 (2018).

11. Chouhan K., Prasad S.B.: Asian J. Pharm. Clin. Res. 9, 314 (2016).

12. Mokomane M., Kasvosve I., de Melo E., Pernica J.M.: Ther. Adv. Infect. Dis. 5, 29 (2018).

13. Zhang Z., Lai S., Yu J., Geng Q., Yang W. et al.: PLoS One 12, 3 (2017).

14. Kampicht S., Pratipanawatr T., Jarernsiripornkul N.: Int. J. Clin. Pharm. 40, 6, (2018).

15. Pirmohamed M., James S., Meakin S., Green C., Scott A.K. el al.: BMJ 329, 15 (2004).

16. Pharmaceutical Law Act of 6 September 2001, No. 45, item 271, art. 37 al., Journal of Laws. 2008.

17. Kieć-Swierzczyńska M., Kręcisz B.: Contact Dermatitis 39, 93 (1998).

18. Shindano A., Marot L., Geubel A.P.: Acta Gastroenterol. Belg. 70, 32 (2007). 
19. IMS Dataview [PRM/8/2018]. Poland Pharmaceutical Dataview - database.

20. Bouree P., Chaput J.C., Krainik F., Michel H, Trépo C.: Gastroenterol. Clin. Biol. 13, 469 (1989).

21. Bouree P., Kouchner G., Ponti M.: Trans. R. Soc. Trop. Med. Hyg. 81, 859 (1987).

22. DuPont H.L., Jiang Z.D., Ericsson C.D., Adachi J.A., Mathewson J.J. et al.: Clin. Infect. Dis. 33, 1809 (2001).

23. Santiago L.T., Ranoa C.P., Chan E.G. et al.: Phil. J. Microbiol. Infect. Dis. 14, 76 (1985).

24. Buisson Y., Larribaud J.: Therapie 44, 123 (1989).

25. Editorial Staff: Towards better patient care: drugs to avoid in 2018. Prescrire International, April 27, 107 (2018).
26. Nifuroxazide: serious immunoallergic reactions. Prescrire International. 268 (2017).

27. https://bi.ema.europa.eu/analyticsSOAP/saw. dll? PortalPages (accessed 21.10.2018).

28. Elloumi-Mseddi J., Msalbi D., Fakhfakh R., Aifa S.: Anticancer Agents Med. Chem. 18 January (2019).

29. Luo Y., Zeng A., Fang A., Song L., Fan C. et al.: Invest. New Drugs (online: 25.01.2019).

30. Jia H., Cui J., Jia X., Zhao J., Feng Y. et al.: Mol. Med. Rep. 16, 9480 (2017).

31. Both L., Botgros R., Cavaleri M.: Euro Surveill. 20, 34 (2015).

Received: 17.12.2018 